\title{
Fast PMI-Based Word Embedding with Efficient Use of Unobserved Patterns
}

\author{
Behrouz Haji Soleimani, ${ }^{1}$ Stan Matwin ${ }^{1,2}$ \\ ${ }^{1}$ Institute for Big Data Analytics, Faculty of Computer Science, Dalhousie University, Halifax NS, Canada \\ ${ }^{2}$ Institute of Computer Science, Polish Academy of Sciences, Warsaw, Poland \\ behrouz.hajisoleimani@dal.ca,stan@cs.dal.ca
}

\begin{abstract}
Continuous word representations that can capture the semantic information in the corpus are the building blocks of many natural language processing tasks. Pre-trained word embeddings are being used for sentiment analysis, text classification, question answering and so on. In this paper, we propose a new word embedding algorithm that works on a smoothed Positive Pointwise Mutual Information (PPMI) matrix which is obtained from the word-word co-occurrence counts. One of our major contributions is to propose an objective function and an optimization framework that exploits the full capacity of "negative examples", the unobserved or insignificant wordword co-occurrences, in order to push unrelated words away from each other which improves the distribution of words in the latent space. We also propose a kernel similarity measure for the latent space that can effectively calculate the similarities in high dimensions. Moreover, we propose an approximate alternative to our algorithm using a modified Vantage Point tree and reduce the computational complexity of the algorithm to $|V| \log |V|$ with respect to the number of words in the vocabulary. We have trained various word embedding algorithms on articles of Wikipedia with 2.1 billion tokens and show that our method outperforms the state-of-the-art in most word similarity tasks by a good margin.
\end{abstract}

\section{Introduction}

Learning continuous representations of words (i.e. word embeddings) are becoming increasingly popular in the machine learning and Natural Language Processing (NLP) community. The goal is to learn a vector space representation for words aiming to capture semantic similarities and syntactic relationships between words. These representations are typically learned from large unlabeled corpora.

Classical vector space models use linear algebraic techniques on the matrix of word-word co-occurrence counts as studied in (Turney and Pantel 2010; Baroni and Lenci 2010). These methods include Latent Semantic Analysis (Deerwester et al. 1990), factorizations of the co-occurrence matrix, factorizations of the Pointwise Mutual Information (PMI) and Positive PMI (PPMI) matrices and so on which can be collectively called as count-based methods. GloVe (Pennington, Socher, and Manning 2014) is certainly

Copyright (c) 2019, Association for the Advancement of Artificial Intelligence (www.aaai.org). All rights reserved. among the most popular word embedding algorithms that directly work on the co-occurrence matrix. It applies a handcrafted weighting scheme in the optimization for each of the word-pair frequencies which is its main advantage over the unweighted optimization in matrix factorization based approaches. Another family of word embedding algorithms uses neural network based approaches to learn the word vectors. (Collobert and Weston 2008) proposed to learn the word embeddings using a feed-forward neural network that predicts a word by looking two words ahead and two words behind. More recently, (Mikolov et al. 2013a) proposed log-bilinear models known as Continuous Bag-OfWords (CBOW) and Skip-Gram to learn continuous representations of words. Lately, contextual word representations derived from bidirectional language models (biLMs) have been shown to improve the performance for many NLP tasks (Peters et al. 2018).

In this paper, we propose a new word embedding algorithm that exploits some information that other algorithms pay little or no attention to. Most word embedding algorithms only use the word pairs that occur in the corpus (i.e. positive examples) and maximize the similarity of those word vectors based on how frequent they co-occur. This can result in a concentration effect: word clusters from totally different topics can be placed somewhat close to each other. There are lots of possible word pairs that never co-occur in the corpus or they co-occur insignificantly, which we call negative examples. We argue that minimizing the similarity of negative examples is also crucial for the quality of the final embedding and results in a better distribution of words in the latent space. Our first major contribution is to design an optimization framework that exploits the full capacity of negative examples in order to push unrelated words away from each other which leads to a better use of the latent space and improves the distribution of words. Skip-Gram with Negative Sampling (SGNS) makes use of the negative examples to a smaller extent in that for each word, it randomly samples $k$ context words as negative examples and minimizes the similarity of the word with those $k$ context words. However, in SGNS, these contexts are employed in an unweighted manner in that they all have the same strength in the optimization. Our second major contribution is that we incorporate a kernelized weighting scheme for the negative examples where their influence in the optimization is propor- 
tionate to their kernel similarity with the word. We show that our kernel similarity measure is a more powerful way of calculating similarities in high-dimensional embeddings where $d>50$ and it enables the algorithm to differentiate between the closer and further points and employ them accordingly. Our third major contribution is that we propose a modified Vantage Point (VP) tree and make it suitable for high dimensional vectors. We then use this VP-tree and propose an approximate solution to our optimization which improves the computational complexity of the method from $|V|^{2}$ to $|V| \log |V|$ with respect to the size of the vocabulary.

We have trained our algorithm as well as several others on the articles of Wikipedia and compared the quality of embeddings on various word similarity and analogy tasks. Results show that our algorithm outperforms the state-ofthe-art in most of the tasks.

\section{Related Work}

Distributed word representation algorithms have been shown to be very effective in capturing certain aspects of similarity between words. Many neural-network based approaches have been proposed for learning distributed word representations (Bengio et al. 2006; Collobert and Weston 2008; Mikolov et al. 2013a; 2013b). Skip-Gram with Negative Sampling (SGNS) (Mikolov et al. 2013a) uses a shallow neural network and trains the network in a way that given a word, it predicts the probability of each context word (Mikolov et al. 2013a). SGNS is still the state-ofthe-art word embedding algorithm and is successfully applied in a variety of linguistic tasks (Mikolov et al. 2013a; $2013 b$ ). Researchers have proposed various modifications to the Skip-Gram model and tried to enrich that with other information. For instance, (Levy and Goldberg 2014a) proposed to use the dependency parsing information and use a dependency-aware context for each word rather than considering all the neighbors in a fixed window. This customization makes the algorithm to learn more from the grammar and less from the semantics. Recently, FastText (Bojanowski et al. 2017), a library developed by Facebook, enriches the Skip-Gram word embeddings with sub-word information. It considers character $n$-grams of different lengths and represents words as the sum of their $n$-gram vectors. This enrichment has been shown to substantially improve the performance of NLP tasks on morphologically rich languages, such as Turkish or Finnish. It is also shown to be very effective for text classification (Joulin et al. 2017). A major advantage of the FastText is that it can handle Out-OfVocabulary (OOV) words by predicting their word vectors based on the learned character $n$-grams embeddings.

Pointwise Mutual Information (PMI) is an information theoretic measure that can be used for finding collocations or associations between words (Church and Hanks 1990) and is widely used in count-based and matrix factorization based word embeddings. For any word pair $\left(w_{i}, w_{j}\right)$, PMI is defined as the log ratio between their joint probability and product of their marginal probabilities:

$$
P M I\left(w_{i}, w_{j}\right)=\log \frac{P\left(w_{i}, w_{j}\right)}{P\left(w_{i}\right) P\left(w_{j}\right)}
$$

Based on the formulation, if two words co-occur more often than being independent then their PMI will be positive, and if they co-occur less frequent than being independent then their PMI will be negative. Since the co-occurrence matrix is sparse, PMI is only calculated on the non-zero entries. Another commonly accepted approach is to use Positive PMI (PPMI) matrix by replacing all the negative values with 0 .

$$
P P M I\left(w_{i}, w_{j}\right)=\max \left(P M I\left(w_{i}, w_{j}\right), 0\right)
$$

In fact, a traditional approach to word representation is to use explicit PPMI representation in which each word is described by its corresponding sparse row vector in the PPMI matrix and it is shown that it outperforms the PMI approach on semantic similarity tasks (Bullinaria and Levy 2007). (Levy and Goldberg 2014b) showed that SGNS is implicitly factorizing a Shifted Positive Pointwise Mutual Information (SPPMI) matrix and they argue that the shift parameter is almost equivalent to the negative sampling parameter $k$ in SGNS. However, their proposed alternative approach using SVD on shifted PPMI matrix provides lower quality embeddings than SGNS mainly because of the unweighted $L_{2}$ optimization in SVD in that frequent and infrequent word pairs have the same amount of influence on the reconstruction error of the matrix. Recently, it is shown that keeping a fraction of small negative PMI values outperforms the PPMI approach (Soleimani and Matwin 2018b). Other than using the raw PPMI matrix or the factorizations of the PPMI matrix, the idea of PMI-based embedding has also been proposed and studied in the literature. (Arora et al. 2016) proposed an objective function similar to that of GloVe which implicitly factorizes the PMI matrix instead of the log co-occurrence matrix. They also showed that if the PMI values are good approximations of the dot products of vectors, then the linear algebraic relations (i.e. word analogies) will hold. Nevertheless, the novelty of our method is in our optimization and the effective use of negative examples.

Another research direction in learning word embeddings is to apply sparsity constraints on the word vectors (Sun et al. 2016; Subramanian et al. 2018) and is shown to improve the quality of embeddings when the desired dimensionality is greater than 300 . For other tips on training good quality embeddings one can refer to (Lai et al. 2016) which study the effect of various hyper-parameters in different embedding algorithms.

\section{Kernelized Unit Ball Word Embedding (KUBWE)}

Our approach at a glance builds a symmetric co-occurrence matrix from the corpus, then calculates an adjusted form of the PMI matrix to remove insignificant and uninformative co-occurrences, and finally obtains the embedding by minimizing a sum of squared error between the PMI values and the cosine similarity of word vectors in the embedded space.

The intuition behind our algorithm is that if two words have a high degree of association (i.e. high PMI), their embedded word vectors must be similar (i.e. high cosine similarity) and if their degree of association is low or zero, they should not be placed close to each other. The second part of 
the intuition is usually ignored in other algorithms and is the main strength of our method.

Our proposed algorithm, KUBWE, is aimed to preserve the word-word connectivity structure (encoded in the PMI matrix) in the final embedding. It uses a spherical representation for the latent space in which points are located on the surface of a hypersphere. The spherical representation is not restrictive as other algorithms also normalize the word vectors to unit length before using them in NLP tasks. Similar spherical embeddings have been proposed and used for visualization (Soleimani and Matwin 2018a) and image clustering application (Soleimani and Matwin 2016).

In the following, we first propose an effective way to measure word-word associations. We then propose our objective function and its gradient descent optimization. Afterward, we propose a kernelized version of the algorithm which enhances the similarity calculations in the latent space. Eventually, we propose a modification to VP-trees to make them suitable for high dimensional data and use them to reduce the computational complexity of the method.

\section{Preparing the Input for the Optimization}

We first calculate the global symmetric word-word cooccurrence counts matrix $X$ by moving an $L$-sized context window over the corpus. This co-occurrence matrix is the main source of information for many word embedding algorithms including GloVe. We also use the same co-occurrence matrix but not in the raw format since many of the cooccurrences are meaningless.

PPMI technique can be used to filter out uninformative co-occurrences. However, a recognized shortcoming of PMI and PPMI is their bias towards infrequent events (Turney and Pantel 2010). This happens when a rare context word $w_{j}$ co-occurs with a word $w_{i}$ a few times (or even once) and this often results in a high PMI value since $P\left(w_{j}\right)$ in PMI's denominator is very small. To overcome this situation, we smooth the distribution of context words in which all context counts are raised to the power of $\alpha$. Hence, we use the following adjusted form of PPMI as input to our optimization:

$$
\begin{gathered}
P P M I_{\alpha}\left(w_{i}, w_{j}\right)=\max \left(P M I \alpha\left(w_{i}, w_{j}\right), 0\right) \\
P M I_{\alpha}\left(w_{i}, w_{j}\right)=\log \frac{P\left(w_{i}, w_{j}\right)}{P\left(w_{i}\right) P_{\alpha}\left(w_{j}\right)} \\
P_{\alpha}\left(w_{j}\right)=\frac{\#\left(w_{j}\right)^{\alpha}}{\sum_{i=1}^{|V|} \#\left(w_{i}\right)^{\alpha}}
\end{gathered}
$$

where $P\left(w_{i}\right)$ and $P_{\alpha}\left(w_{j}\right)$ are the unsmoothed and smoothed distribution of words. Context distribution smoothing alleviates PMI's bias towards rare words, like other smoothing techniques (Pantel and Lin 2002; Turney and Littman 2003). It enlarges the probability of a rare context $P\left(w_{j}\right)$, which in turn reduces the PMI of $\left(w_{i}, w_{j}\right)$ for any $w_{i}$ co-occurring with the rare context $w_{j}$.

We refer to the adjusted PPMI matrix as $A$ with entries $a_{i j}=P P M I_{\alpha}\left(w_{i}, w_{j}\right)$. In all our experiments, we used $\alpha=0.75$ which is known to be a good smoothing factor (Mikolov et al. 2013b).

\section{Cost Function}

Our proposed method uses a spherical representation for the latent space which is common for natural language processing tasks. In this representation, we use cosine similarity as our similarity measure between word vectors:

$$
S\left(\vec{w}_{i}, \vec{w}_{j}\right)=\frac{\vec{w}_{i} \cdot \vec{w}_{j}}{\left\|\vec{w}_{i}\right\|\left\|\vec{w}_{j}\right\|}
$$

The objective function is then defined to minimize the sum of squared differences between the smoothed PPMI values $a_{i j}$ and the similarities in the embedded space:

$$
\mathcal{J}(W)=\frac{1}{2} \sum_{i=1}^{|V|} \sum_{j=1}^{|V|}\left(a_{i j}-S\left(\vec{w}_{i}, \vec{w}_{j}\right)\right)^{2}
$$

where $a_{i j}$ and $S\left(\vec{w}_{i}, \vec{w}_{j}\right)$ are calculated using equations (3) and (6), respectively. Here we propose a constrained variant of the objective function as follows which makes the normalizations fade away as well as resulting in a simplified gradient:

$$
\begin{gathered}
\mathcal{J}(W)=\frac{1}{2} \sum_{i=1}^{|V|} \sum_{j=1}^{|V|}\left(a_{i j}-\vec{w}_{i} \cdot \vec{w}_{j}\right)^{2} \\
\text { subject to }: \vec{w}_{i} \cdot \vec{w}_{i}=1 \forall i
\end{gathered}
$$

Here, the objective function forces the word vectors to be on the surface of a hypersphere. The unit length constraints is not restrictive since the surface of a hypersphere has only one fewer degree of freedom than the entire volume. Therefore, the surface will have enough room to locate all the points in the desired way. In the constrained form of the objective function, the dot product of output vectors gives the cosine similarity and is used as the similarity measure between word vectors in the embedded space.

Cosine values range in $[-1,+1]$ and we normalize them to $[0,+1]$ by using $\frac{1}{2}\left(\vec{w}_{i} \cdot \vec{w}_{j}+1\right)$. This is done because $a_{i j}$ values are also positive. In fact, PMI values $a_{i j}$ can be greater than 1, however, we handle this in the next section. By normalizing the cosine values to $[0,+1]$, if the PMI of two words is high (i.e. significant co-occurrence), then the method tries to place them as close as possible. And if the PMI is zero (i.e. unobserved or insignificant co-occurrence), $a_{i j}=0$, then the method tries to put them as far as possible.

\section{Optimization}

We use stochastic gradient descent to minimize our objective function. We initialize the word vectors randomly on the surface of a hypersphere and incrementally change the configuration of them to get a better objective value. We have used the general projected gradient descent framework (Lin 2007) in order to satisfy the constraints. In this setting, the solution is projected onto the feasible region after each iteration. The gradient of the cost function with respect to a word 
vector $\vec{w}_{i}$ is:

$$
\begin{aligned}
\nabla \mathcal{J}\left(\vec{w}_{i}\right)=\frac{\partial \mathcal{J}}{\partial \vec{w}_{i}} & =\sum_{j=1}^{|V|}\left[-a_{i j} \vec{w}_{j}+\left(\vec{w}_{i} \cdot \vec{w}_{j}\right) \vec{w}_{j}\right] \\
& =-\overbrace{\sum_{j=1}^{|V|} a_{i j} \vec{w}_{j}+\sum_{j=1}^{v_{1}}\left(\vec{w}_{i} \cdot \vec{w}_{j}\right) \vec{w}_{j}}^{v_{2}}
\end{aligned}
$$

The gradient consists of two components $v_{1}$ and $v_{2}$. The former, $v_{1}$, defines the sum of attractive forces being applied to the word vector while the latter, $v_{2}$, defines the sum of repulsive forces being applied to the word vector. In fact, each context word is having a contribution in the gradient of $\vec{w}_{i}$. Since $W$ is a sparse affinity matrix, only the nonzero PMIs will have a contribution in the attractive force $v_{1}$. However, all other words vectors will have a contribution to the repulsive force $v_{2}$ based on their similarity to the word vector being tuned. In other words, $\vec{w}_{i}$ will be attracted to its significant context vectors and it will be pushed away from its current neighbors if it shouldn't be close to them. Please note that, by normalizing the similarities in $[0,+1], v_{2}$ will be the sum of weighted repulsive forces applied from different word vectors.

One of the distinguishing characteristics of our method is that unlike SGNS and GloVe which update a word vector based on each entry in the matrix (or each occurrence of two words in the moving window), our algorithm updates the word vector $\vec{w}_{i}$ based on its entire row in the smoothed PMI matrix $A$. This way, all the attractive forces in $v_{1}$ are automatically weighted according to their smoothed PMI value. Therefore, using an auxiliary weighting function as in GloVe is totally unnecessary and here, the weighting is done seamlessly. As for the negative force $v_{2}$, we take out the nonzero PMIs and calculate the negative force only based on the word pairs with zero PMI. This slightly improves the distribution of words as each context will have either an attractive or repulsive effect when updating a particular word vector.

The time complexity for calculating the gradient vector for a particular word $\vec{w}_{i}$ is $O(|V| \times d)$ where $|V|$ and $d$ are the vocabulary size and the dimensionality of the embedded space, respectively. The calculation of the repulsive force is more costly than the attractive force because of the similarity computations. In total, the optimization requires $O\left(i \times|V|^{2} \times d\right)$ time, where $i$ is the number of iterations in the optimization. However, the size of the vocabulary $|V|$ is several orders of magnitude less than the number of tokens in the corpus. For instance, in our experiments on Wikipedia (dump of March 2016), 163,188 words were extracted from 2.1 billion tokens. Later, we will adopt an approximation technique to reduce the time complexity.

\section{Kernelized Objective Function}

Figure 1 illustrates the distribution of cosine similarities between thousands of random vectors in different dimensionality. We generated 100,000 pairs of random vectors in each case and calculated their cosine similarity and plotted the distribution of similarities with respect to the dimensionality

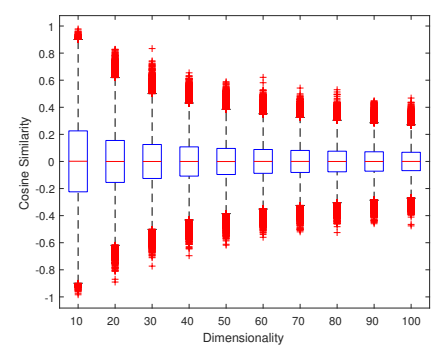

Figure 1: Distribution of cosine similarity of 100,000 pairs of random vectors. The distribution of cosine similarities is $\mathcal{N}\left(0, \frac{1}{\sqrt{d}}\right)$.

of vectors. As we can see from the figure, in lower dimensions we have a wider distribution between $[-1,+1]$. But, as we increase the dimensionality, the distribution narrows down and the chances of getting two similar or dissimilar vectors is getting lower and lower. In fact, in higher dimensions, almost all vectors will be equidistant and almost orthogonal to each other. Specialized distance measures have been proposed in the literature (Soleimani, Matwin, and De Souza 2015), but the curse of dimensionality in metric spaces is not well-studied.

Considering ineffectiveness of cosine similarity and every other metric in higher dimensions, we propose a kernelized variant of our objective function which improves the distribution of similarities and enables the algorithm to differentiate between the closer and further vectors.

Looking at equation (9), the repulsive force $v_{2}$ consists of a dot product of vectors in the embedded space which measures their similarity. Here, we can apply a kernel to calculate the similarities of vectors in an implicit highdimensional feature space. If $\phi(\cdot)$ is the implicit mapping function to the high-dimensional space, the kernel function $K: \mathbb{R}^{d} \times \mathbb{R}^{d} \rightarrow \mathbb{R}$ will compute the inner product of those vectors in an efficient way $K\left(\vec{w}_{i}, \vec{w}_{j}\right)=\left\langle\phi\left(\vec{w}_{i}\right), \phi\left(\vec{w}_{j}\right)\right\rangle$. The gradient of the objective function is then:

$$
\frac{\partial \mathcal{J}}{\partial \vec{w}_{i}}=-\sum_{j=1}^{|V|} a_{i j} \vec{w}_{j}+\sum_{j=1}^{|V|} K\left(\vec{w}_{i}, \vec{w}_{j}\right) \times \vec{w}_{j}
$$

Here we apply the kernel just in the repulsive force and not in the objective function directly. PMI has proven to be able to capture the strength of association of words very well (Bullinaria and Levy 2007; Church and Hanks 1990). Therefore, we do not need to adjust the attractive force and we only want to tune the similarities in the embedded space. Moreover, applying the kernel in the repulsive force will simplify the formulation and consequently, simplify the numerical optimization by preventing the derivative of $\phi($.$) to$ appear in the gradient. Here, we propose to use a polynomial kernel to adjust the nonlinearity and further strengthen the effect of closer points in negative force.

$$
K\left(\vec{w}_{i}, \vec{w}_{j}\right)=\left(\vec{w}_{i} \cdot \vec{w}_{j}+1\right)^{p}
$$

where $p$ is the degree of the polynomial kernel. Please note that in the kernelized form, there is no need to normalize the 
dot product between $[0,+1]$ because of the increment in the formula which makes all similarities positive. In fact, by using a polynomial kernel $p \geq 2$, the negative cosine similarities are weakened while the positive cosine similarities are strengthened. This provides a more powerful similarity measure for higher dimensions and more discriminative power for our learning algorithm.

\section{Reducing the time complexity by approximating the repulsive force}

The most time consuming part of our algorithm is the calculation of repulsive force that requires all the pairwise similarity calculations. Here, we propose an alternative solution and reduce the complexity from $|V|^{2}$ to $|V| \log |V|$ with respect to the size of the vocabulary. In the fast version of the algorithm we only take into account the $k$ nearest neighbors of each word for calculating the repulsive force $v_{2}$ in equation (9). This way, each word $w_{i}$ attracts words $w_{j}$ if $a_{i j}>0$ (i.e. positive PMI) and pushes away words $w_{j}$ that are among its nearest neighbors $w_{j} \in k n n\left(w_{i}\right)$. In fact, we do not need to push words further away if they are already far apart, but if they are mistakenly close to the word, we use the repulsive force to improve the distribution of words. Please note that the nearest neighbors for each word are selected from the negative set with $a_{i j}=0$. This is achieved using a binary search in the positive indices to check whether the neighbor has positive PMI or not.

We use a Vantage Point tree (VP-tree) (Yianilos 1993) combined with a heap data structure to calculate and maintain the nearest neighbors of each word. A VP-tree is a binary tree that is hierarchically built by randomly selecting a point as a vantage point and calculating the distance from the vantage point to every other point. Then using the median distance as the splitting threshold, half of the points fall under the left child and the other half fall under the right child. The splitting process for each node using the median distance can be thought of centering a hyper-sphere on the vantage point in a way that half of the point are inside the hyper-sphere and the other half are outside of it.

VP-trees along with $k d$-trees (Toth, O'Rourke, and Goodman 2017) and almost any other data structure suffer from the curse of dimensionality and are only suitable for lowdimensional data. In fact, in high dimensions all the points will be almost equidistant. Therefore, while searching a query point in a VP-tree, if it falls within the hypersphere of a vantage point we still need to check the outside points since the query will be very close to the median distance and the nearest neighbor may be on the other side. And similarly if the query point falls outside of the hypersphere of the vantage point we still need to check the inside points. Consequently, this leads to traversing the entire tree and its performance is no better than the exhaustive search.

There exist many approximate nearest neighbor search methods as summarized in (Muja and Lowe 2014). However, due to the nature of our problem and its known structure we propose our own alternative. In our algorithm, all the words are distributed on the surface of a hypersphere and therefore, their dot product is equivalent to their cosine similarity. Cosine similarity of vectors in $d$ dimensions has a distribution of $\mathcal{N}\left(0, \frac{1}{\sqrt{d}}\right)$ (Spruill and others 2007). Similarly, the cosine distances are distributed from $\mathcal{N}\left(1, \frac{1}{\sqrt{d}}\right)$. For instance, considering $d=100$, then the cosine distances will be in $[0.7,1.3]$ range with 99.7 probability (i.e. $\mu \pm 3 \sigma$ ). We incorporate this information in our VP-tree search in order to decide whether or not the other branch needs visiting. Using this technique we get more than $99 \%$ accuracy on our $k$ nearest neighbor search while ensuring the $\log |V|$ search time for each word.

By adopting the aforementioned technique, the time complexity of our algorithm is $O(i d|V|(\bar{p}+(k+\log |V|) \log \bar{p}))$ where $\bar{p}$ is the average number of positive examples per word $(\bar{p} \ll|V|)$ and $k$ is the number of nearest neighbors (i.e. negative examples) that is used. $O(i d|V| \bar{p})$ correspond to the attractive force calculations while $O(i d|V|(k+\log |V|) \log \bar{p})$ correspond to the repulsive force calculations. $\log \bar{p}$ correspond to the binary search inside the positive indices to ensure that the negative set do not overlap with the positives.

KUBWE is implemented in C using the OpenMP parallel computing library and the source code can be found on GitHub $^{1}$.

\section{Experiments}

We have used all the articles of English Wikipedia (dump of March 2016) as the training corpus which has around 2.1 billion tokens after applying a few basic preprocessing steps. As for the vocabulary, we have limited the vocabulary to English words by using the WordNet database which resulted in about $163 \mathrm{~K}$ words. In our experiments, all the algorithms were trained on the exact same preprocessed input corpus to ensure a fair comparison. We have also used the exact same vocabulary for all the algorithms.

For the quantitative evaluation of algorithms we have used two well-known tasks of word similarity and word analogy. For the word similarity task, there exist several datasets containing word pairs with their corresponding humanassigned similarity score. In this task we have used 8 different dataset including WordSim353 (WS-ALL), WordSim Similarity (WS-SIM) and WordSim Relatedness (WS-REL), $M E N$, SimLex, MC, RG, and Stanford Rare Words (RWSTN). For the analogy task, we have used Google's analogy dataset (Mikolov et al. 2013a) which contains 19,544 questions of the form " $a$ is to $a^{*}$ as $b$ is to $b^{*}$ ". Given three of the words, the algorithm is expected to predict the fourth word. About half of the questions are semantic (e.g. "father is to son as mother is to daughter") and the other half are syntactic questions (e.g. "big is to bigger as tall is to taller").

\section{Analysis of the Polynomial Kernel Degree}

We first analyze the effect of the kernel degree in a fixed dimensionality of 100 . Our algorithm is trained using various polynomial degrees $1,3, \ldots, 21$ and the quality of embeddings is measured on different word similarity and word analogy tasks. Figure 2 shows the performances with respect to the kernel degree. As we can see from Figures $2 \mathrm{a}$

\footnotetext{
${ }^{1}$ https://github.com/behrouzhs/kubwe
} 


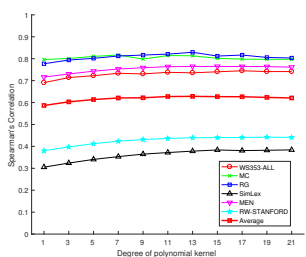

(a) Word similarity task

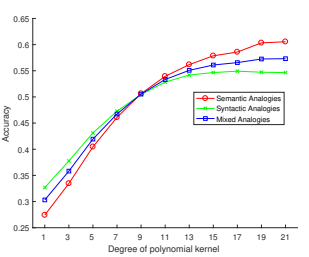

(b) Word analogy task
Figure 2: Quality of embeddings $(d=100)$ obtained from KUBWE using different kernel degrees measured on different (a) word similarity, and (b) word analogy tasks.

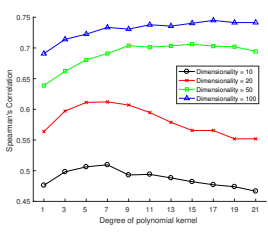

(a) WordSim 353

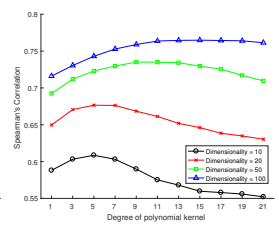

(b) MEN

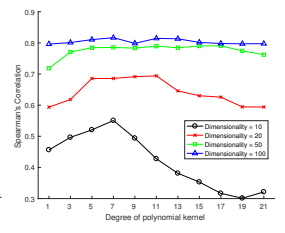

(c) $\mathrm{MC}$
Figure 3: The effect of polynomial kernel degree in different dimensionality in KUBWE algorithm evaluated on (a) WordSim353, (b) MEN, (c) MC.

and $2 \mathrm{~b}$ the general trend is increasing as we increase the degree of the polynomial. This shows that in 100-dimensional space using a high degree polynomial kernel significantly improves the distribution of words, nonetheless, it reaches a plateau at some point.

In another experiment, we run the algorithm with different embedding dimensionality $(10,20,50$, and 100) and in each case, we use various kernel degrees $1,3, \ldots, 21$. Figure 3 illustrates the performance of our algorithm on different dimensionality using different kernel degrees. As we can see from the figures, generally we get better embedding as we increase the dimensionality of the embedding. This is true in other algorithms as well. We can also observe that in lower dimensions (10 and 20), using a high degree kernel will degrade the quality of embedding significantly. This is because of distribution of cosine similarities (i.e. dot products) in the first place. If we look back at Figure 1 we see that when $d=10$ and $d=20$ the similarities are spread all over [-1, $+1]$. Using a high degree polynomial in such cases causes a few negative examples to have extremely high kernel similarity with the word being updated and they dominate all the rest of the negative examples. This leads to inappropriate use of negative examples which in turn deteriorates the quality of the embedding. However, in higher dimensions where the distributions of similarities are close to zero (almost orthogonal vectors), using a higher degree polynomial will further improve the similarity calculations in the repulsive force.

\section{Quantitative Evaluation}

Table 1 compares 14 algorithms on 8 word similarity datasets. The numbers in the table are Pearson's correlation between the rankings provided by the algorithms and the rankings of the human-scoring. These algorithms are

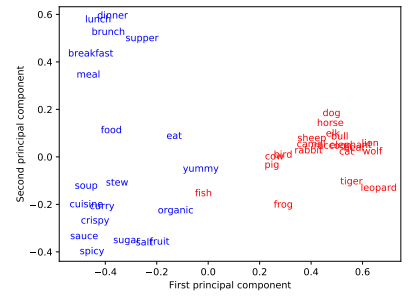

(a) KUBWE

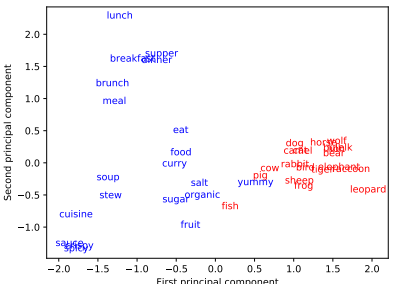

(b) SGNS
Figure 4: Distribution of 40 word vectors from two groups of 20 animal names and 20 food related words. PCA algorithm is applied on 100-dimensional vectors from KUBWE (left) and SGNS (right) to obtain a 2-d visualization.

selected mainly because of their popularity and the reproducibility/availability of their source code. SVD, SVD-Log, and SVD-Sqrt are the factorizations of the co-occurrence, the $\log$ co-occurrence, and the square root of co-occurrence matrices, respectively. SVD-PPMI and SVD-SPPMI are the SVD factorization of the PPMI and Shifted PPMI (with shift parameter of $-\log 5$ ) matrices, respectively. SVD-NS is the factorization of thresholded PMI table which incorporates a fraction of negative PMI values (Soleimani and Matwin $2018 \mathrm{~b})$. GloVe is trained with its recommended parameter setting (i.e. $x_{\max }=100$ ). FastText is trained with the recommended parameter settings that considers character $\mathrm{n}$ grams of length 3 to 6 . CBOW and SGNS are trained with negative sampling set to 5 and 10 . Our proposed algorithm, KUBWE, is trained with $p=13$, and the fast KUBWE is trained with $k=3000$. The dimensionality of embeddings is 100 in the top part of the table and 300 in the bottom 5 rows.

As we can see from Table 1, our algorithm provides the best results on 7 out of 8 datasets using 100-dimensional embeddings and on 6 out of 8 datasets using 300-dimensional embeddings. It is noteworthy to mention that even the fast approximate version of KUBWE outperforms the state-ofthe-art in 6 out of 8 word similarity tasks. Using 100dimensional embeddings, SGNS is the best on only WordSim Similarity dataset, and on the rest of the datasets, our method outperforms others by a good margin. We have to mention that in the analogy task GloVe provides the best results and is better than KUBWE and SGNS. However, GloVe's performance on word similarity tasks is not comparable with that of KUBWE and SGNS.

\section{Qualitative Evaluation}

Figure 4 illustrates the 2-d distribution of 40 word vectors (20 animal names and 20 food related words) obtained by applying Principal Components Analysis (PCA) on the resulting embedding vectors from KUBWE and SGNS. As we can see, our algorithm provides a better separation and a larger gap between the two word clusters by pushing unrelated words away from each other which is the consequence of the repulsive force and better utilization of negative exam- 
Table 1: Evaluation of different word embedding algorithms on 8 word similarity datasets. The dimensionality of the embeddings is 100 for the top part and 300 for the bottom 5 rows. Numbers in the table are Pearson's rank-order correlation between the human scores and scores from algorithms.

\begin{tabular}{lcccccccc}
\hline & WS-SIM & WS-REL & WS-ALL & MC & RG & MEN & SimLex & RW-STN \\
\# of word pairs & 203 & 252 & 353 & 30 & 65 & 3000 & 999 & 2034 \\
\hline SVD & 0.533 & 0.282 & 0.410 & 0.331 & 0.491 & 0.390 & 0.202 & 0.229 \\
SVD-Sqrt & 0.754 & 0.605 & 0.681 & 0.729 & 0.667 & 0.657 & 0.286 & 0.395 \\
SVD-Log & 0.741 & 0.629 & 0.699 & 0.783 & 0.693 & 0.712 & 0.328 & 0.386 \\
SVD-PPMI & 0.720 & 0.638 & 0.692 & 0.803 & 0.740 & 0.740 & 0.318 & 0.381 \\
SVD-SPPMI & 0.669 & 0.593 & 0.646 & 0.774 & 0.709 & 0.716 & 0.297 & 0.360 \\
GloVe $\left(x_{m}=100\right)$ & 0.674 & 0.553 & 0.599 & 0.664 & 0.706 & 0.704 & 0.315 & 0.329 \\
CBOW $(k=10)$ & 0.740 & 0.584 & 0.665 & 0.703 & 0.756 & 0.709 & 0.326 & 0.393 \\
CBOW $(k=5)$ & 0.745 & 0.585 & 0.671 & 0.742 & 0.773 & 0.707 & 0.327 & 0.398 \\
FastText & 0.765 & 0.649 & 0.713 & 0.793 & 0.787 & 0.741 & 0.326 & 0.442 \\
SGNS $(k=10)$ & $\mathbf{0 . 7 7 4}$ & 0.650 & 0.712 & 0.801 & 0.789 & 0.732 & 0.324 & 0.423 \\
SGNS $(k=5)$ & 0.758 & 0.651 & 0.709 & 0.794 & 0.783 & 0.730 & 0.323 & 0.421 \\
SVD-NS $(\alpha=-2.5)$ & 0.752 & 0.654 & 0.712 & 0.801 & 0.752 & 0.753 & 0.330 & 0.399 \\
Fast KUBWE & 0.746 & 0.663 & 0.728 & 0.805 & 0.807 & 0.735 & 0.367 & $\mathbf{0 . 4 4 4}$ \\
KUBWE $(p=13)$ & 0.770 & $\mathbf{0 . 6 9 2}$ & $\mathbf{0 . 7 4 0}$ & $\mathbf{0 . 8 0 9}$ & $\mathbf{0 . 8 2 7}$ & $\mathbf{0 . 7 6 1}$ & $\mathbf{0 . 3 7 6}$ & 0.439 \\
\hline GloVe $\left(x_{m}=100\right)$ & 0.695 & 0.572 & 0.621 & 0.749 & 0.744 & 0.726 & 0.354 & 0.353 \\
FastText & $\mathbf{0 . 7 9 4}$ & 0.669 & 0.733 & 0.821 & 0.805 & 0.766 & 0.381 & $\mathbf{0 . 4 8 3}$ \\
SGNS $(k=10)$ & 0.792 & 0.667 & 0.732 & 0.830 & 0.799 & 0.753 & 0.383 & 0.455 \\
Fast KUBWE & 0.781 & 0.704 & 0.753 & 0.828 & 0.836 & 0.765 & $\mathbf{0 . 4 2 1}$ & 0.451 \\
KUBWE $(p=13)$ & 0.783 & $\mathbf{0 . 7 1 0}$ & $\mathbf{0 . 7 5 9}$ & $\mathbf{0 . 8 5 1}$ & $\mathbf{0 . 8 4 5}$ & $\mathbf{0 . 7 7 5}$ & 0.411 & 0.452 \\
\hline
\end{tabular}

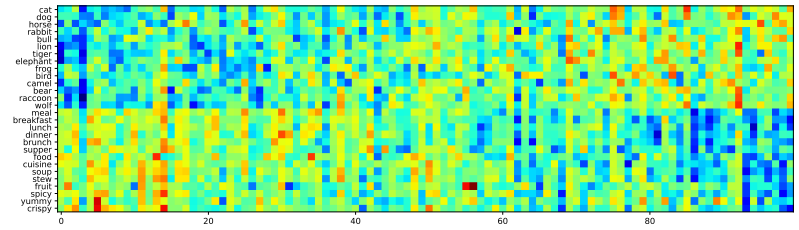

(a) KUBWE

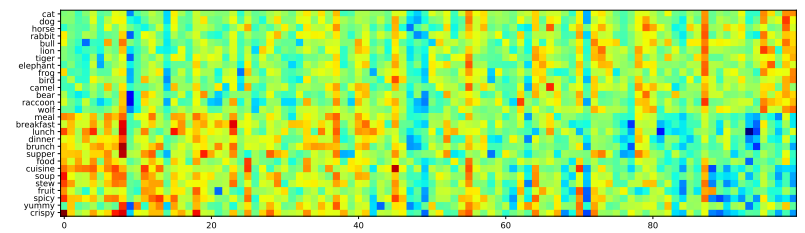

(b) SGNS

Figure 5: Heatmaps of 28 word vectors obtained from KUBWE (top) and SGNS (bottom). The top 14 rows in the heatmaps are animal names and the bottom 14 rows are food related words.

ples. Moreover, in the SGNS distribution "yummy" is closer to the animal group which is not correct, and "salt" and "organic" are also very close to the boundary.

Figure 5 shows the heatmaps of 28 word vectors (14 animal names at the top and 14 food related words at the bottom) from the KUBWE and SGNS embeddings. In each of the heatmaps, columns are ordered by the difference between the average magnitude of the features among the two word groups. Here we can see that our algorithm provides a better inter-group dissimilarity and a nicer distinction between two unrelated word groups. This property will poten- tially improve the accuracy of classifiers in many NLP tasks including text classification.

\section{Conclusion}

In this paper, we analyzed different word embedding algorithms and proposed our algorithm KUBWE. Our method has clear advantages over matrix factorization methods since the attractive and repulsive forces in the optimization are weighted according to the similarities in the input (i.e. smoothed PPMI) and similarities in the output (i.e. polynomial kernel), respectively. It has also advantages over "prediction-based" methods such as SGNS and GloVe for two main reasons: 1) The smoothed PPMI input to our algorithm is more reliable than the raw co-occurrence counts. 2) The adaptive way of utilizing the negative examples prevents the concentration effect and improves the distribution of words in the final embedding. Moreover, the effect of cosine similarity in higher dimensions is analyzed and a kernelized way of calculating similarities is suggested to alleviate the ineffectiveness of cosine similarity. Furthermore, by adopting a modified VP-tree and approximating the repulsive force in the optimization we reduced the computational complexity of our algorithm by orders of magnitude. Our algorithm has only one parameter which is the polynomial degree $p$ in the exact version and the number of negative neighbors $k$ in the fast approximate version. As a rule of thumb, one should pick the degree proportionate to the log of embedding dimensionality $p \approx \log d$, and the number of negative neighbors roughly equal to the square root of the number of words in the vocabulary $k \approx \sqrt{|V|}$. Further theoretical analysis and a more systematic approach to choosing the kernel parameter will be investigated in the future work. 


\section{References}

Arora, S.; Li, Y.; Liang, Y.; Ma, T.; and Risteski, A. 2016. A latent variable model approach to pmi-based word embeddings. Transactions of the Association for Computational Linguistics 4:385-399.

Baroni, M., and Lenci, A. 2010. Distributional memory: A general framework for corpus-based semantics. Computational Linguistics 36(4):673-721.

Bengio, Y.; Schwenk, H.; Senécal, J.-S.; Morin, F.; and Gauvain, J.-L. 2006. Neural Probabilistic Language Models. Berlin, Heidelberg: Springer Berlin Heidelberg. 137-186.

Bojanowski, P.; Grave, E.; Joulin, A.; and Mikolov, T. 2017. Enriching word vectors with subword information. Transactions of the Association for Computational Linguistics 5:135-146.

Bullinaria, J. A., and Levy, J. P. 2007. Extracting semantic representations from word co-occurrence statistics: A computational study. Behavior research methods 39(3):510526.

Church, K. W., and Hanks, P. 1990. Word association norms, mutual information, and lexicography. Computational linguistics 16(1):22-29.

Collobert, R., and Weston, J. 2008. A unified architecture for natural language processing: Deep neural networks with multitask learning. In Proceedings of the 25th international conference on Machine learning, 160-167. ACM.

Deerwester, S.; Dumais, S. T.; Furnas, G. W.; Landauer, T. K.; and Harshman, R. 1990. Indexing by latent semantic analysis. Journal of the American society for information science 41(6):391.

Joulin, A.; Grave, E.; Bojanowski, P.; and Mikolov, T. 2017. Bag of tricks for efficient text classification. In Proceedings of the 15th Conference of the European Chapter of the Association for Computational Linguistics, volume 2, 427-431.

Lai, S.; Liu, K.; He, S.; and Zhao, J. 2016. How to generate a good word embedding. IEEE Intelligent Systems 31(6):514.

Levy, O., and Goldberg, Y. 2014a. Dependency-based word embeddings. In 52nd Annual Meeting of the Association for Computational Linguistics, volume 2, 302-308.

Levy, O., and Goldberg, Y. 2014b. Neural word embedding as implicit matrix factorization. In Advances in neural information processing systems, 2177-2185.

Lin, C.-J. 2007. Projected gradient methods for nonnegative matrix factorization. Neural computation 19(10):27562779.

Mikolov, T.; Chen, K.; Corrado, G.; and Dean, J. $2013 a$. Efficient estimation of word representations in vector space. arXiv preprint arXiv:1301.3781.

Mikolov, T.; Sutskever, I.; Chen, K.; Corrado, G. S.; and Dean, J. 2013b. Distributed representations of words and phrases and their compositionality. In Advances in Neural Information Processing Systems, 3111-3119.

Muja, M., and Lowe, D. G. 2014. Scalable nearest neighbor algorithms for high dimensional data. IEEE transactions on pattern analysis and machine intelligence 36(11):22272240.

Pantel, P., and Lin, D. 2002. Discovering word senses from text. In Proceedings of the eighth ACM SIGKDD international conference on Knowledge discovery and data mining, 613-619. ACM.

Pennington, J.; Socher, R.; and Manning, C. D. 2014. Glove: Global vectors for word representation. In EMNLP, volume 14, 1532-1543.

Peters, M.; Neumann, M.; Yih, W.-t.; and Zettlemoyer, L. 2018. Dissecting contextual word embeddings: Architecture and representation. In Empirical Methods in Natural Language Processing, EMNLP.

Soleimani, B. H., and Matwin, S. 2016. Nonlinear dimensionality reduction by unit ball embedding (ube) and its application to image clustering. In 15th IEEE International Conference on Machine Learning and Applications, ICMLA 2016, 983-988. IEEE.

Soleimani, B. H., and Matwin, S. 2018a. Dimensionality reduction and visualization by doubly kernelized unit ball embedding. In Advances in Artificial Intelligence: 31 st Canadian Conference on Artificial Intelligence, Canadian AI 2018, 224-230. Springer.

Soleimani, B. H., and Matwin, S. 2018b. Spectral word embedding with negative sampling. In Thirty-Second AAAI Conference on Artificial Intelligence, AAAI 2018.

Soleimani, B. H.; Matwin, S.; and De Souza, E. N. 2015. A density-penalized distance measure for clustering. In Advances in Artificial Intelligence: 28th Canadian Conference on Artificial Intelligence, Canadian AI 2015, 238-249. Springer.

Spruill, M., et al. 2007. Asymptotic distribution of coordinates on high dimensional spheres. Electronic communications in probability 12:234-247.

Subramanian, A.; Pruthi, D.; Jhamtani, H.; BergKirkpatrick, T.; and Hovy, E. 2018. Spine: Sparse interpretable neural embeddings. In $A A A I$ Conference on Aritificial Intelligence, AAAI.

Sun, F.; Guo, J.; Lan, Y.; Xu, J.; and Cheng, X. 2016. Sparse word embeddings using 11 regularized online learning. In Proceedings of the Twenty-Fifth International Joint Conference on Artificial Intelligence, IJCAI.

Toth, C. D.; O'Rourke, J.; and Goodman, J. E. 2017. Handbook of discrete and computational geometry. Chapman and Hall/CRC.

Turney, P. D., and Littman, M. L. 2003. Measuring praise and criticism: Inference of semantic orientation from association. ACM Transactions on Information Systems (TOIS) 21(4):315-346.

Turney, P. D., and Pantel, P. 2010. From frequency to meaning: Vector space models of semantics. Journal of artificial intelligence research 37:141-188.

Yianilos, P. N. 1993. Data structures and algorithms for nearest neighbor search in general metric spaces. In SODA, volume 93, 311-321. 